\title{
Internal Efficiency Analysis of 280 nm Light Emitting Diodes
}

\author{
J. Piprek, ${ }^{i}$ C. Moe, S. Keller, S. Nakamura, and S. P. DenBaars \\ Solid-State Lighting and Display Center, Materials Department \\ University of California, Santa Barbara, CA 93106
}

\begin{abstract}
Compact ultraviolet light sources are currently of high interest for a range of applications, including solid-state lighting, short-range communication, and bio-chemical detection. We report on the design and analysis of AlGaN-based lightemitting diodes with an emission wavelength near $280 \mathrm{~nm}$. Internal device physics is investigated by threedimensional numerical simulation. The simulation incorporates a drift-diffusion model for the carrier transport, built-in polarization, the wurtzite energy band-structure of strained quantum wells, as well as radiative and nonradiative carrier recombination. Critical material parameters are identified and their impact on the simulation results is investigated. Limitations of the internal quantum efficiency by electron leakage and nonradiative recombination are analyzed. Increasing the stopper layer bandgap is predicted to improve the quantum efficiency and the light output of our LED substantially.
\end{abstract}

Keywords: Ultraviolet light source, light-emitting diode, LED, AlGaN quantum wells, internal quantum efficiency, current leakage, numerical simulation

\section{INTRODUCTION}

Compact ultraviolet (UV) light sources are currently of high interest for applications in solid-state lighting, short-range communication, and bio-chemical detection. Prime candidates are AlGaN light-emitting diodes (LEDs). However, the output power of such devices is still below the values achieved in the visible wavelength range. ${ }^{1}$ We therefore utilize three-dimensional (3D) physics-based simulation to better understand internal device physics. Previously, we investigated UV LEDs with an emission wavelength near $340 \mathrm{~nm}$, obtaining good agreement with measurements. ${ }^{2,3}$ The same simulation procedure is here applied to new devices with an emission wavelength near $280 \mathrm{~nm}{ }^{4}$ The measured external quantum efficiency of these devices is about $0.1 \%$, i.e., only one out of 1000 injected electrons contributes a photon to the measured output power. This paper reveals the physical mechanisms behind this low efficiency. The device structure is described in Section 2, followed by an outline of the theoretical model and a discussion of material parameters in Section 3. Main simulation results are presented and analyzed in Section 4.

\section{DEVICE STRUCTURE}

The target emission wavelength of $280 \mathrm{~nm}$ requires the use of an active region with relatively high aluminum mole fraction. Our multi-quantum well (MQW) active region consists of four $3 \mathrm{~nm}$ thick $\mathrm{Al}_{0.41} \mathrm{Ga}_{0.59} \mathrm{~N}$ quantum wells and five $7 \mathrm{~nm}$ thick $\mathrm{Al}_{0.5} \mathrm{Ga}_{0.5} \mathrm{~N}$ barriers grown on a thick n-doped $\mathrm{Al}_{0.64} \mathrm{Ga}_{0.36} \mathrm{~N}$ layer. Note that this MQW structure is lattice matched to $\mathrm{Al}_{0.64} \mathrm{Ga}_{0.36} \mathrm{~N}$ and not to $\mathrm{GaN}$ as in our previous studies. A 20 -nm p-doped $\mathrm{Al}_{0.65} \mathrm{Ga}_{0.35} \mathrm{~N}$ electron stopper layer is grown on top of the MQW, followed by a 10-nm p-GaN contact layer. Mesas of various sizes are formed by reactive ion etching. The simulations are for $300 \mu \mathrm{m}$ x $300 \mu \mathrm{m}$ LEDs with an U-shaped n-contact as shown schematically in Fig. 1. The vertical layer structure is given in Tab. 1, together with estimated values for the effective

${ }^{i}$ Corresponding author, e-mail: piprek@ieee.org 
free carrier density resulting from Si doping (n) and Mg doping (p), respectively. Due to the high activation energy of the $\mathrm{Mg}$ acceptor in $\mathrm{AlGaN}$, the free hole density is substantially smaller than the $\mathrm{Mg}$ doping density.

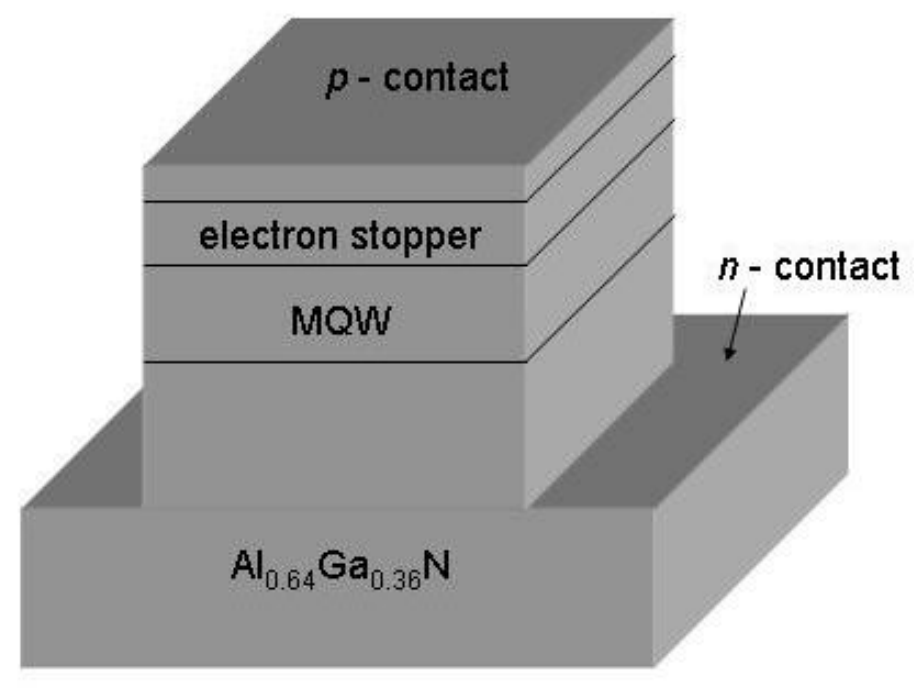

Fig. 1: Schematic view of our top-emitting LED with U-shaped $n$-contact.

\begin{tabular}{|l|r|l|}
\hline Material & $\begin{array}{r}\mathbf{d} \\
{[\mathbf{n m}]}\end{array}$ & $\begin{array}{l}\mathbf{n}, \mathbf{p} \\
{\left[\mathbf{1 0}^{\mathbf{1 8}} \mathbf{c m}^{-3}\right]}\end{array}$ \\
\hline $\mathrm{p}-\mathrm{GaN} \mathrm{10}$ & 1.0 \\
\hline $\mathrm{p}-\mathrm{Al}_{0.65} \mathrm{Ga}_{0.35} \mathrm{~N}$ & 20 & 0.0001 \\
\hline $\mathrm{n}-\mathrm{Al}_{0.50} \mathrm{Ga}_{0.50} \mathrm{~N}$ & 7 & 0.01 \\
\hline $\mathrm{i}-\mathrm{Al}_{0.41} \mathrm{Ga}_{0.59} \mathrm{~N}$ & 3 & - \\
\hline $\mathrm{n}-\mathrm{Al}_{0.50} \mathrm{Ga}_{0.50} \mathrm{~N}$ & 7 & 4.0 \\
\hline $\mathrm{i}-\mathrm{Al}_{0.41} \mathrm{Ga}_{0.59} \mathrm{~N}$ & 3 & - \\
\hline $\mathrm{n}-\mathrm{Al}_{0.50} \mathrm{Ga}_{0.50} \mathrm{~N}$ & 7 & 4.0 \\
\hline $\mathrm{i}-\mathrm{Al}_{0.41} \mathrm{Ga}_{0.59} \mathrm{~N}$ & 3 & - \\
\hline $\mathrm{n}-\mathrm{Al}_{0.50} \mathrm{Ga}_{0.50} \mathrm{~N}$ & 7 & 4.0 \\
\hline $\mathrm{i}-\mathrm{Al}_{0.41} \mathrm{Ga}_{0.55} \mathrm{~N}$ & 3 & - \\
\hline $\mathrm{n}-\mathrm{Al}_{0.50} \mathrm{Ga}_{0.50} \mathrm{~N}$ & 7 & 4.0 \\
\hline $\mathrm{n}-\mathrm{Al}_{0.64} \mathrm{Ga}_{0.36} \mathrm{~N}$ & 250 & 17.0 \\
\hline
\end{tabular}

Tab. 1: Epitaxial layer structure (d - thickness; $\mathrm{n}, \mathrm{p}$ - carrier concentration from doping; i - not intentionally doped).

\section{THEORETICAL MODEL AND MATERIAL PARAMETERS}

We employ the simulation software APSYS ${ }^{5}$ which self-consistently combines the 3D simulation of carrier transport, heat flux, quantum well effects, and optical ray tracing. The transport model included drift and diffusion of electrons and holes, Fermi statistics, built-in polarization and thermionic emission at hetero-interfaces, as well as spontaneous and 
SRH recombination of carriers. For the quantum wells, Schrödinger and Poisson equations are solved iteratively to account for the quantum well deformation with changing device bias. Spontaneous emission of photons by electron-hole recombination within the quantum well is calculated by a free carrier model including the wurtzite energy band structure. Self-heating is neglected here since it becomes irrelevant with proper LED mounting and packaging. Further details of the model can be found elsewhere. ${ }^{3,6}$

An important issue in any device simulation is the selection of appropriate values for the various material parameters employed in the simulation. ${ }^{3,7}$ As published values for many parameters vary substantially in the literature, we shall investigate the impact of several parameters that are crucial for our simulation results:

i. Conduction band offset $\left(\Delta E_{c}\right)$ : The conduction band offset at AlGaN hetero-interfaces is the most critical material parameter in our simulation as small changes can strongly alter the electron leakage from the MQW. ${ }^{3}$ Recent calculations give a conduction band offset ratio $\Delta \mathrm{E}_{\mathrm{c}} / \Delta \mathrm{E}_{\mathrm{g}}$ between 0.66 and 0.81 for the $\mathrm{AlN} / \mathrm{GaN}$ interface. ${ }^{8}$ While using an offset ratio of 0.7 for most of our hetero-interfaces, ${ }^{7}$ we will vary this number at the important interface between MQW barrier and stopper layer. The room-temperature AlGaN band gap is calculated as $\mathrm{E}_{\mathrm{g}}(\mathrm{x})=\mathrm{x}(6.13 \mathrm{eV})+(1-\mathrm{x})(3.42 \mathrm{eV})-\mathrm{x}(1-\mathrm{x})(1.5 \mathrm{eV})$ with the Al mole fraction $\mathrm{x}{ }^{9}$

ii. $\quad$ Nonradiative carrier lifetime $\left(\tau_{S R H}\right)$ : Nonradiative Shockley-Read-Hall (SRH) recombination is governed by the type and density of crystal defects, which depends on the fabrication process. While quantum well SRH carrier lifetimes near $1 \mathrm{~ns}$ are often assumed, ${ }^{2}$ nonradiative carrier lifetimes below 100 ps have been reported for AlGaN layers at room temperature. ${ }^{10}$ In the next section, we therefore investigate two cases with $1 \mathrm{~ns}$ and $0.1 \mathrm{~ns}$ lifetime, respectively.

iii. Polarization charge $\left(\sigma_{f i x}\right)$ : Built-in interface charges due to spontaneous and piezoelectric polarization are known to influence the performance of nitride devices. ${ }^{11}$ Table 2 lists the fixed charges calculated for our interfaces using the non-linear formulas from Fiorentini et al. ${ }^{12}$ However, our experimental investigation of quantum well thickness variations indicates only small polarization effects, possibly caused by partial compensation of polarization charges by charged interface defects. We therefore multiply the charges listed in Tab. 2 by a factor of 0.5 in some of our simulations. ${ }^{13}$

\begin{tabular}{|l|l|l|}
\hline Interface & Charge type & Charge density \\
\hline $\mathrm{Al}_{0.64} \mathrm{Ga}_{0.36} \mathrm{~N} / \mathrm{Al}_{0.50} \mathrm{Ga}_{0.50} \mathrm{~N}$ & negative & $8.37 \times 10^{12} \mathrm{~cm}^{-2}$ \\
\hline $\mathrm{Al}_{0.50} \mathrm{Ga}_{0.50} \mathrm{~N} / \mathrm{Al}_{0.41} \mathrm{Ga}_{0.59} \mathrm{~N}$ & negative & $5.01 \times 10^{12} \mathrm{~cm}^{-2}$ \\
\hline $\mathrm{Al}_{0.41} \mathrm{Ga}_{0.59} \mathrm{~N} / \mathrm{Al}_{0.50} \mathrm{Ga}_{0.50} \mathrm{~N}$ & positive & $5.01 \times 10^{12} \mathrm{~cm}^{-2}$ \\
\hline $\mathrm{Al}_{0.50} \mathrm{Ga}_{0.50} \mathrm{~N} / \mathrm{Al}_{0.65} \mathrm{Ga}_{0.35} \mathrm{~N}$ & positive & $9.22 \times 10^{12} \mathrm{~cm}^{-2}$ \\
\hline $\mathrm{Al}_{0.65} \mathrm{Ga}_{0.35} \mathrm{~N} / \mathrm{GaN}$ & negative & $32.41 \times 10^{12} \mathrm{~cm}^{-2}$ \\
\hline
\end{tabular}

Table 2: Fixed polarization charges as calculated for lattice constants matched to $\mathrm{Al}_{0.64} \mathrm{Ga}_{0.36} \mathrm{~N}$.

\section{SIMULATION RESULTS AND ANALYSIS}

Figure 2 shows a 3D plot of the local spontaneous recombination rate as calculated for $100 \mathrm{~mA}$ injection current. The internal photon generation is strongest along the sidewalls with adjacent n-contact. This is attributed to current crowding caused by the low conductivity of p-doped regions. ${ }^{2}$ To study internal device physics in more detail, we plot vertical profiles near the corners of the U-shaped contact in the following.

Figure 3 gives the MQW band diagram for the two different polarization cases. Built-in polarization causes a significant deformation of the quantum wells that separates electrons and holes within the wells. Consequently, radiative emission rate, quantum efficiency, and output power are reduced by the polarization. With full polarization, the calculated internal quantum efficiency is $\eta_{\text {int }}=0.04 \%$, i.e., only 4 out of 10,000 electrons generate a photon in the quantum wells. 
Since the number of externally measured photons is always lower than the number of photons generated inside the LED, the external quantum efficiency is $\eta_{\mathrm{ext}}<0.01 \%$ in the simulation. This number is an order of magnitude below the measured value, which indicates that at least one of our default material parameters is not appropriate for our device $\left(\Delta \mathrm{E}_{\mathrm{c}}=310 \mathrm{meV}, \tau_{\mathrm{SRH}}=1 \mathrm{~ns}, \sigma_{\mathrm{fix}}=100 \%\right)$. Table 3 summarizes the effect of parameter variations in the simulation.

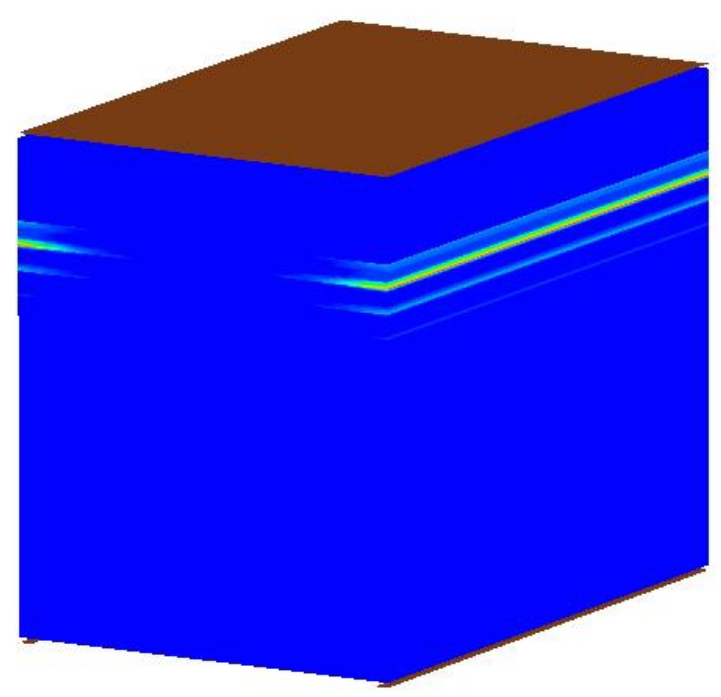

Fig. 2: 3D plot of the internal photon emission rate. The n-contact is below the region shown and the middle part of the U-shaped metal lies behind the back surface (cf. Fig. 1).

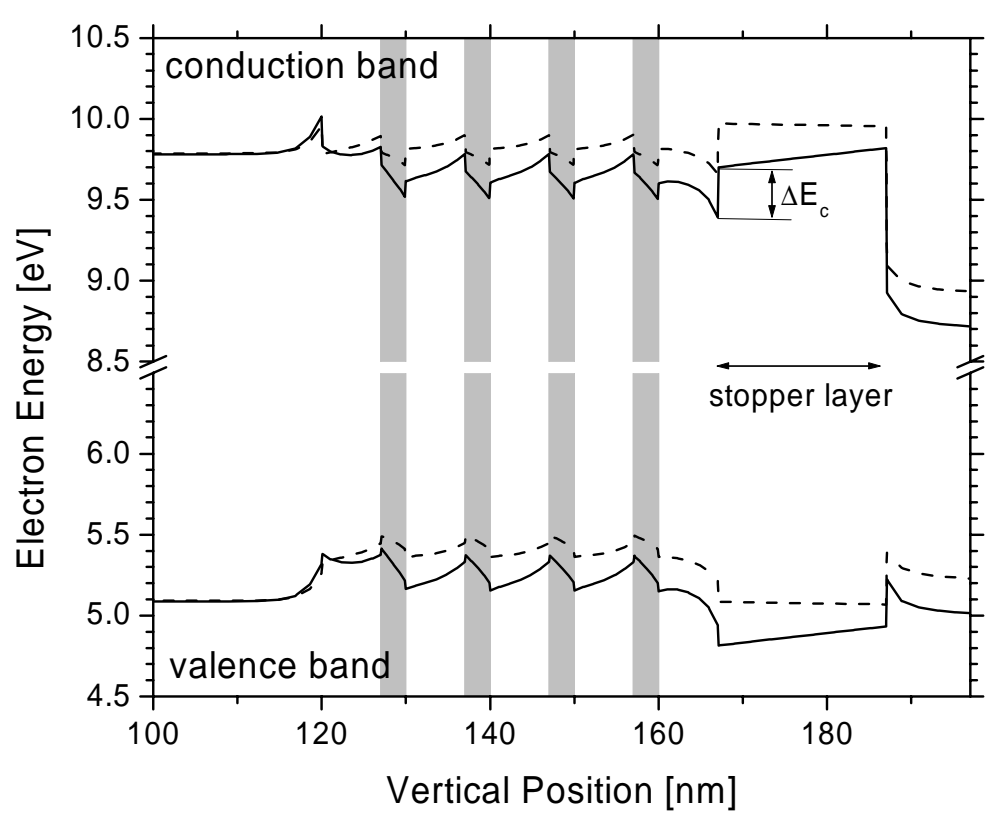

Fig. 3 Energy band diagram of the active region with $100 \%$ (solid) and 50\% polarization (dashed). The conduction band offset ratio is 0.7 at all interfaces with $\Delta \mathrm{E}_{\mathrm{c}}=310 \mathrm{meV}$ for the stopper layer $\left(\tau_{\mathrm{SRH}}=1 \mathrm{~ns}\right)$. Quantum wells are marked by the grey areas. 


\begin{tabular}{|l|l|l|l|l|}
\hline Case & $\boldsymbol{\tau}_{\text {SRH }}$ & $\Delta \mathbf{E}_{\mathbf{c}}$ & $\boldsymbol{\sigma}_{\text {fix }}$ & $\boldsymbol{\eta}_{\text {int }}$ \\
\hline $\mathrm{a}$ & $1 \mathrm{~ns}$ & $310 \mathrm{meV}$ & $100 \%$ & $0.04 \%$ \\
\hline $\mathrm{b}$ & $1 \mathrm{~ns}$ & $310 \mathrm{meV}$ & $50 \%$ & $0.15 \%$ \\
\hline $\mathrm{c}$ & $1 \mathrm{~ns}$ & $350 \mathrm{meV}$ & $100 \%$ & $0.53 \%$ \\
\hline $\mathrm{d}$ & $1 \mathrm{~ns}$ & $350 \mathrm{meV}$ & $50 \%$ & $1.92 \%$ \\
\hline $\mathrm{e}$ & $0.1 \mathrm{~ns}$ & $350 \mathrm{meV}$ & $100 \%$ & $0.05 \%$ \\
\hline $\mathrm{f}$ & $0.1 \mathrm{~ns}$ & $350 \mathrm{meV}$ & $50 \%$ & $0.17 \%$ \\
\hline $\mathrm{g}$ & $0.1 \mathrm{~ns}$ & $310 \mathrm{meV}$ & $100 \%$ & $0.01 \%$ \\
\hline $\mathrm{h}$ & $0.1 \mathrm{~ns}$ & $310 \mathrm{meV}$ & $50 \%$ & $0.02 \%$ \\
\hline
\end{tabular}

Tab. 3: Summary of parameter variations and their impact on the internal quantum efficiency $\eta_{\text {int }}\left(\tau_{\mathrm{SRH}}-\right.$ nonradiative carrier lifetime, $\Delta \mathrm{Ec}$ - stopper layer band offset, $\sigma_{\mathrm{fix}}$ - built-in polarization).

In case $b$ of Tab. $3,50 \%$ reduction of the polarization charges results in the internal efficiency $\eta_{\text {int }}=0.15 \%$ which is attributed to a better overlap of electron and hole wavefunction in the quantum well as well as to a larger effective energy barrier imposed by the stopper layer. With a uniform offset ratio of 0.7 , the conduction band offset between MQW barrier and stopper layer is $\Delta \mathrm{E}_{\mathrm{c}}=310 \mathrm{meV}$, however, the high density of fixed positive charges at that interface causes significant band bending which pulls down the barrier (see Fig. 3) and allows for electrons to leak into the p-side of the device. Figure 4 plots the vertical electron current density across the active region. With the default value $\Delta \mathrm{E}_{\mathrm{c}}=$ $310 \mathrm{meV}$, most of electrons leak into the p-side where they recombine with holes, thus diminishing hole injection into the MQW (dashed lines). By increasing the offset to $\Delta \mathrm{E}_{\mathrm{c}}=350 \mathrm{meV}$, which corresponds to an offset ratio of 0.8 , the electron leakage from the MQW is reduced, allowing for more holes to enter the active region. With the enlarged energy barrier, the internal quantum efficiency is increased to $0.53 \%$ for full polarization and to $1.92 \%$ for half the polarization (cases $\mathrm{c}$ and $\mathrm{d}$ in Tab. 3).

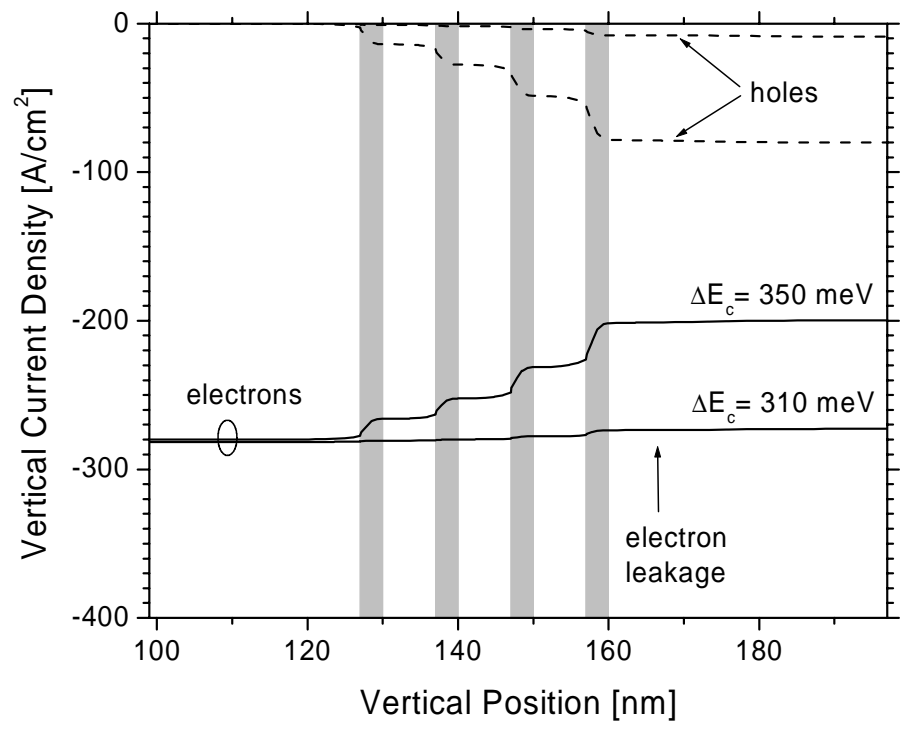

Fig. 4: Vertical profile of the current density for electrons (solid) and holes (dashed) for two different conduction band offsets of the stopper layer $\left(100 \%\right.$ polarization, $\left.\tau_{\mathrm{SRH}}=1 \mathrm{~ns}\right)$. 


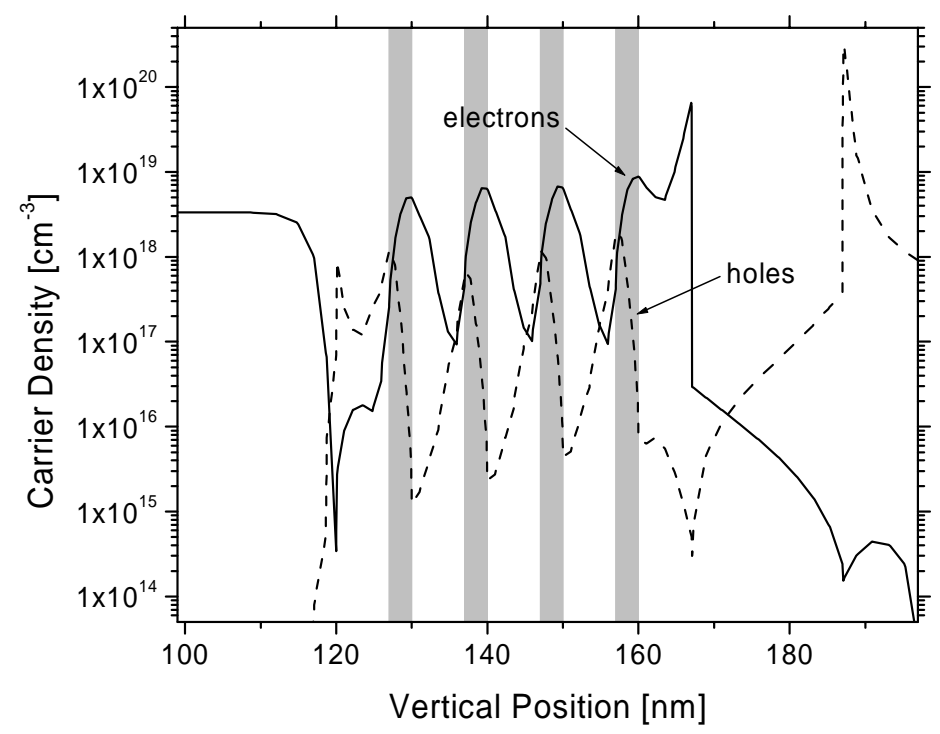

Fig. 5: Vertical profile of the carrier density of electrons (solid) and holes (dashed) for $100 \%$ polarization, $\Delta \mathrm{E}_{\mathrm{c}}=350 \mathrm{meV}$, and $\tau_{\mathrm{SRH}}=1 \mathrm{~ns}$.

For $\Delta \mathrm{E}_{\mathrm{c}}=350 \mathrm{meV}$, Fig. 5 plots the carrier density profiles at $100 \mathrm{~mA}$ injection current. The built-in polarization separates electrons and holes in the quantum wells. The quantum well electron concentration is above $5 \times 10^{18} \mathrm{~cm}^{-3}$, whereas the hole concentration is below $2 \times 10^{18} \mathrm{~cm}^{-3}$, due to the poor hole injection indicated in Fig. 4. The hole density peaks on the $\mathrm{p}$-side of the stopper layer as expected from the large number of negative polarization charges there (cf. Tab. 2). The electron density peaks on the other side of the stopper layer. In both cases, the minority carrier density is very small leading to negligible carrier recombination at both stopper layer interfaces.

The corresponding vertical profile of the radiative recombination rate is plotted in Fig. 6 (solid line). It is maximum within the quantum wells. Hardly any radiation is generated within the p-doped regions, despite the strong electron leakage. Note that defect related photon emission mechanisms are not considered in our simulation. Radiative recombination competes with SRH recombination which may be underestimated by the uniform nonradiative carrier lifetime of $1 \mathrm{~ns}$ assumed thus far. The dashed line in Fig. 6 gives the photon emission rate for $\tau_{\mathrm{SRH}}=0.1 \mathrm{~ns}$ and it reveals a strong impact of the defect density. The quantum well emission now exhibits a steep decline from the $\mathrm{p}$-side to the $\mathrm{n}$ side. Carriers mainly recombine via defects and hardly any holes reach the n-side quantum well. As SRH recombination is enhanced by an order of magnitude, the internal quantum efficiency drops from $0.53 \%$ to $0.05 \%$ (cases $\mathrm{c}$ and $\mathrm{e}$ in Tab. 3). The SRH recombination rate is profiled in Fig. 7 for both cases. It is significantly stronger than the photon emission rate, even in the quantum wells. In the case with the shorter SRH lifetime (dashed), the nonradiative recombination strongly declines across the quantum wells as less and less holes are available.

The remaining cases f-h in Tab. 3 give an unrealistically low internal efficiency, indicating that the assumption of an uniformly short SRH carrier lifetime of $0.1 \mathrm{~ns}$ is not appropriate for our device. Experimental lifetime studies are needed to establish more realistic numbers for our different AlGaN layers. However, it seems even more important to measure the conduction band offset $\Delta \mathrm{E}_{\mathrm{c}}$ between $\mathrm{MQW}$ barrier layer and $\mathrm{Al}_{0.65} \mathrm{Ga}_{0.35} \mathrm{~N}$ stopper layer as this parameter shows the largest impact on the internal quantum efficiency in Tab. 3. Increasing the $\mathrm{Al}$ mole fraction of this layer will most certainly enhance the quantum efficiency and the output power of our LED. 


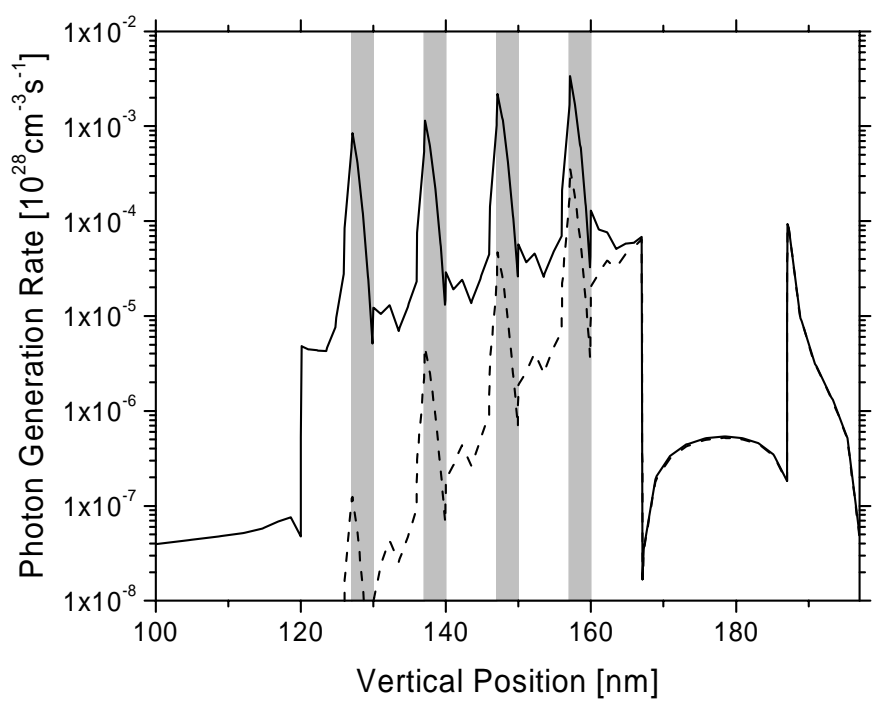

Fig. 6: Vertical profile of the photon generation rate for $\tau_{\mathrm{SRH}}=1 \mathrm{~ns}$ (solid) and $\tau_{\mathrm{SRH}}=0.1 \mathrm{~ns}$ (dashed). Full polarization is assumed here with $\Delta \mathrm{E}_{\mathrm{c}}=350 \mathrm{meV}$.

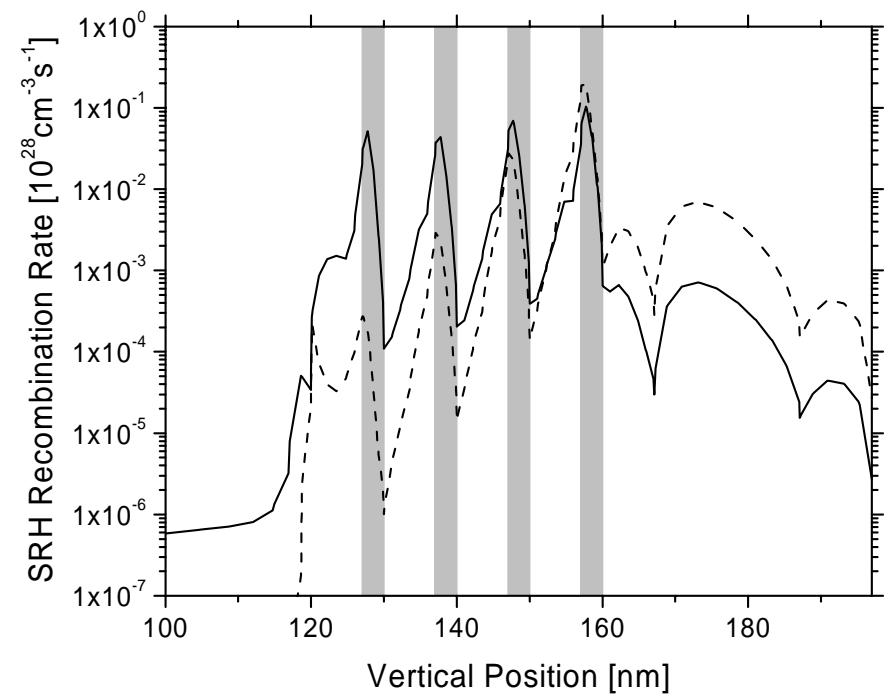

Fig. 7: Vertical profile of the nonradiative recombination rate for $\tau_{\mathrm{SRH}}=1 \mathrm{~ns}$ (solid) and $\tau_{\mathrm{SRH}}=0.1 \mathrm{~ns}$ (dashed) with $100 \%$ polarization and $\Delta \mathrm{E}_{\mathrm{c}}=350 \mathrm{meV}$. 


\section{SUMMARY}

The internal device physics of $280 \mathrm{~nm} \mathrm{AlGaN/GaN} \mathrm{LEDs} \mathrm{is} \mathrm{analyzed} \mathrm{by} \mathrm{advanced} \mathrm{3D} \mathrm{simulation.} \mathrm{Electron} \mathrm{leakage} \mathrm{into}$ the p-doped region as well as nonradiative recombination are found to strongly limit the light output power. The internal quantum efficiency is calculated for several combinations of key material parameters, showing a major impact of stopper layer band offset, nonradiative recombination lifetime, and built-in polarization.

\section{REFERENCES}

1 W. H. Sun, J. P. Zhang, V. Adivarahan, A. Chitnis, M. Shatalov, S. Wu, V. Mandavilli, J. W. Yang, and M. A. Khan, "AlGan-based $280 \mathrm{~nm}$ light-emitting diodes with CW powers in excess of 1.5mW" Appl. Phys. Lett., vol. 85, pp. 531-534, 2004.

2 Joachim Piprek, Tom Katona, Steven P. DenBaars, and Simon Li, "3D Simulation and Analysis of AlGaN/GaN Ultraviolet Light Emitting Diodes," in: Light-Emitting Diodes: Research, Manufacturing and Applications VIII, S.Stockman, H. W. Yao, and E. F. Schubert (eds.), SPIE Proc. 5366, pp. 127-136, 2004.

3 J. Piprek and S. Li, "GaN-based Light Emitting Diodes," Chapter 10 in: Optoelectronic Devices - Advanced Simulation and Analysis, J. Piprek (ed.), Springer Verlag, New York, 2004.

4 C. Moe, P. Cantu, H. Masui, K. Vampola, S. Keller, S. P. DenBaars, C. Hussell and D. Emerson, "280 nm Deep Ultraviolet LEDs Grown on SiC," to be published in J. Crystal Growth, 2004.

5 APSYS 2004.08, Crosslight Software, Burnaby, Canada (http://www.crosslight.com).

6 J. Piprek, Semiconductor Optoelectronic Devices - Introduction to Physics and Simulation, Academic Press, San Diego, 2003

7 I. Vurgaftman and J. R. Meyer, " Band parameters for nitrogen-containing semiconductors,” J. Appl. Phys., vol. 94, pp. $3675-3691,2003$.

8 C. G. Van de Walle and J. Neugebauer, "Universal alignment of hydrogen levels in semiconductors, insulators, and solutions," Nature, vol. 423, pp. 626-628, 2003.

9 S. Nikishin, G. Kipshidze, V. Kuryatkov, K. Choi, I `u. Gherasoiu, L. Grave de Peralta, A. Zubrilov V. Tretyakov, K. Copeland, T. Prokofyeva, M. Holtz, R. Asomoza, Yu. Kudryavtsev, and H. Temkin, "Gas source molecular beam epitaxy of high quality AlGaN on Si (111),”J. Vac. Sci. Technol. B, vol. 19, pp. 1409-1412, 2001.

10 T. Onuma, S. F. Chichibu, et al., "Radiative and nonradiative processes in strain-free AlGaN films studied by timeresolved photoluminescence and positron annihilation techniques," J. Appl. Phys., vol. 95, pp. 2495-2504, 2004.

11 J. Piprek, "Simulation of GaN-based Light Emitting Devices," in: Simulation of Semiconductor Processes and Devices (ed. G. Wachutka and G. Schrag), Springer Verlag, Wien, 2004.

12 V. Fiorentini, F. Bernardini, and O. Ambacher, "Evidence for nonlinear macroscopic polarization in III-V nitride alloy heterostructures," Appl. Phys. Lett., vol. 80, pp. 1204-1206, 2002.

13 C. A. Flory and G. Hasnain, "Modeling of GaN Optoelectronic Devices and Strain-Induced Piezoelectric Effects," IEEE J. Quantum Electronics, vol. 37, pp. 244 - 253, 2001. 